\title{
ACTIVE FILTERS AND HYBRID TECHNOLOGY
}

\author{
P. L. MORAN \\ Department of Electronic and Electrical Engineering, University of Technology, Loughborough, Leicestershire
}

(Received August 20, 1979; in final form January 14, 1980)

\begin{abstract}
The conditions under which the choice of circuit used for the active filter is important are discussed with relationship to both the technology used and the application of the circuit. A number of common circuits are critically analysed and compared with respect to these criteria.
\end{abstract}

\section{INTRODUCTION}

The subject of active filters has been discussed for a number of years. Sallen and Key ${ }^{1}$ were probably the people who initiated the extensive interest in the subject, although earlier work has been mentioned. ${ }^{2}$ The importance of Sallen and Key's work is difficult to assess; it was their paper that demonstrated that general filter functions could be implemented in practice using only amplifiers, resistors and capacitors; although probably the most important aspect of this paper was that it introduced the concept of cascading low sensitivity second order sections, rather than attempting to manufacture the entire filter in one stage. They also presented a number of second order circuits some of which are still in use today. Probably the next paper that initiated a new area of research came in 1966, 13 years later, when Orchard ${ }^{3}$ reasoned that filters based on a structure with minimum insertion loss would have low sensitivity. Such structures are based on Darlington's ${ }^{4}$ insertion loss synthesis technique and classically require the use of inductors. The search was on therefore for a satisfactory method of inductance simulation. Various methods were proposed of which Antoniou's ${ }^{5}$ general impedance converter was probably the most successful. However simply replacing the inductors by simulated inductance does not necessarily lead to an economical circuit since capacitor tolerances in the $0.1 \%$ to $1 \%$ region are frequently required for such filters and narrow tolerance components are very expensive. These subjects will be returned to later.

If an abstract search is carried out using the general index "active filters" a vast number of papers will be found. Over the last few years they have been published at the rate of well over one hundred a year. It is of interest therefore to ask why it is that over twenty five years since the subject was first introduced so much research is still going on, much of it on circuits that look remarkably similar to those first presented by Sallen and Key. The answer to this question probably lies in the differing boundary conditions imposed in part by the various applications of active filters, and also by changing technologies with which the filters may be constructed. One important design of active filter is a high order band pass filter with a relatively narrow bandwidth built to the highest standards of performance and reliability. In such circumstances it is necessary to use designs with the lowest sensitivity, components with the highest stability and a packaging technique that maximises the reliability. Another important design is a medium order low pass filter with a cut off frequency of a few kilohertz. Whilst reliability is important, since the potential market for such a design runs into many hundreds of thousands per annum, the most important factor is cost. Research work therefore centres on circuit theory that enables filter functions to be met with less sensitive designs, designs that require less stable components, designs that use fewer of the expensive components (even if more of the less expensive components are required), designs that are easier to manufacture and adjust, and technology that improves the stability and reliability of the components and is less expensive to manufacture. At the high performance end of the specifications, there is probably little choice in the design of the filter and manufacturing techniques used and Saraga et $\mathrm{al}^{19,20}$ have recently published details of some developments of such high 
performance filters. However, at the lower performance end, the optimum solution will be a compromise between conflicting requirements both in terms of the circuit and the technology used.

There are essentially three technologies available to the engineer for manufacturing filters. Conventional printed wiring assemblies using discrete components appear to offer the cheapest solution based on a simple component count. However even modest filter designs require quite narrow component tolerances. Narrow tolerance components are expensive, so too are adjustable resistors which could be used to overcome both the resistor and capacitor tolerances and to compensate for the "strays". While thick or thin film hybrid assemblies are rather more expensive, they do allow through the use of laser trimming, at no extra cost, resistors of any value (within a certain range) and further the resistors may be adjusted with the circuit functioning. In addition the hybrid approach can, by employing "chip" amplifiers and capacitors, provide a low weight, low volume, high reliability assembly, with the only major problem being how to package the resulting module. The third possible approach is to use an integrated circuit. In order to make an analogue filter, both high value, high stability resistors and capacitors are necessary. Such components are not really feasible on integrated circuits, although some manufacturers are now incorporating nichrome resistors on integrated circuits and laser trimming has been reported.

However, with the recent advances in microprocessor technology and as many of the lower performance filters are used with digital systems, there is the possibility that digital filters may take over at the lower frequency end of the spectrum. However such a "chip" will be large and hence could be quite expensive, although it will be rather easier to package than a hybrid. It will be interesting to see how such a technique performs, particularly with regard to dynamic range and upper frequency limit. $\dagger$

It can be seen therefore that at the current time probably the optimum solution for active filters is a hybrid approach, and for the high performance filters is likely to remain so for some considerable time. At the lower performance end, digital filtering techniques will probably begin to make some impact in the near future. It is important therefore that the best low pass filter design is chosen and hence a low pass filter will be used as the basis for comparison of conflicting requirements in the remainder of this paper.

$\dagger$ It will also be interesting to see how monolithic switched capacitor techniques progress.

\section{TECHNOLOGY CONSIDERATIONS}

Probably the most important economic consideration in any hybrid construction is the packaging technique used. The cost of a metal hermetic package suitable for encapsulating a substrate, say $1.0^{\prime \prime}$ by $0.6^{\prime \prime}$, is often considerably more than the cost of the hybrid components assembled on the substrate. In this situation therefore small economies in the design are not of any great significance and it is probably sensible to use the one with the greatest tolerance to component variations, irrespective of the number of components required. However if the application allows a low cost plastic encapsulation to be used then the particular circuit chosen is of great importance especially with regard to the component tolerance and stability required and ease of functional adjustment.

One of the more important cost considerations in an active filter is the capacitors used. Generally it is necessary to use high stability NPO chip components, but, unfortunately the range and tolerance of these devices is strictly limited. Figure 1 shows the approximate relative cost of chip capacitors as a function of value, while Figure 2 gives a guide to the

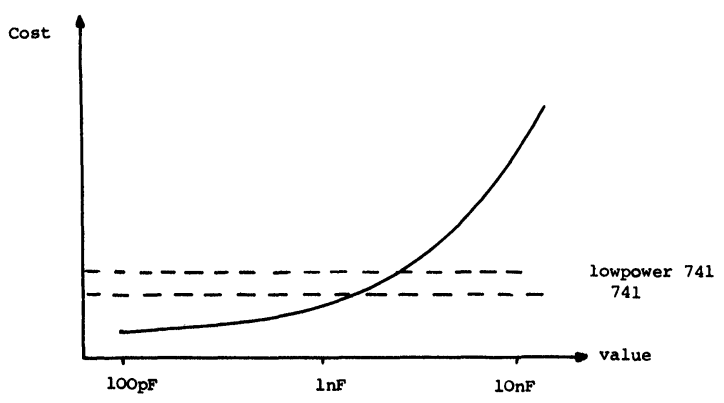

FIGURE 1 Approximate cost of 10\% NPO "chip" capacitors vs capacitance value.

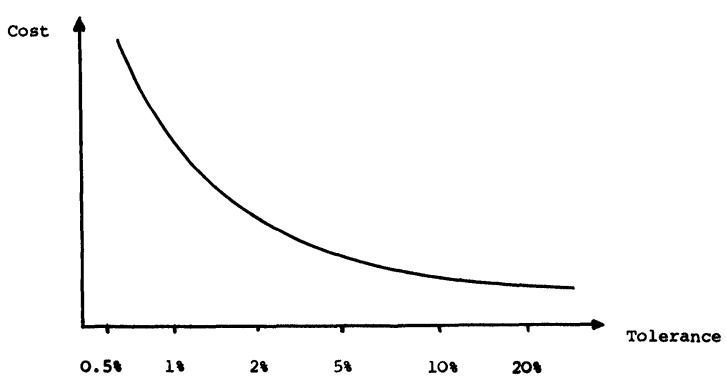

FIGURE 2 Approximate cost of $1 \mathrm{nF}$ NPO "chip" capacitor vs tolerance. 
TABLE I

Typical resistor and capacitor parameters for hybrid microcircuits.

\begin{tabular}{llll}
\hline & Thick Film & Thin Film & Chip NPO Capacitors \\
\hline $\begin{array}{l}\text { Range } \\
\text { Temperature }\end{array}$ & $1 \Omega / \square \rightarrow 10 \mathrm{M} \Omega / \square$ & $50 \Omega / \square \rightarrow 300 \Omega / \square$ & $1 \mathrm{pF} \rightarrow 0.01 \mu \mathrm{F}$ \\
$\begin{array}{l}\text { Coefficient } \\
\begin{array}{l}\text { Differential } \\
\text { Temperature }\end{array}\end{array}$ & $50 \rightarrow 100 \mathrm{ppm}$ & $5 \rightarrow 15 \mathrm{ppm}$ & $30 \mathrm{ppm}$ \\
$\begin{array}{l}\text { Coefficient } \\
\begin{array}{l}\text { Stability } \\
\text { (typical) }\end{array}\end{array}$ & $5 \rightarrow 10 \mathrm{ppm}$ & $1 \rightarrow 5 \mathrm{ppm}$ & \\
$\begin{array}{l}\text { Tolerance } \\
\text { (economical) }\end{array}$ & $0.5 \%$ & $\sim 0.2 \%$ & very low \\
\hline
\end{tabular}

way in which the cost varies as a function of component tolerance for a $1 \mathrm{nF}$ device. Also shown on Figure 1 is the approximate relative value of a 741 amplifier. These figures need to be treated with some caution as they are liable to change from time to time and also the actual cost of assembling an amplifier depends very much on the techniques used. (For instance a quad amplifier chip may be assembled with approximately the same effort as a single amplifier chip particularly when using automatic bonding techniques. However it may prove more difficult to arrange for the larger number of conductor tracks to be brought to the chip and in the event of an amplifier failure all four amplifiers must be replaced.)

Typical parameters of the capacitors and resistors used in thick and thin film technology are given in Table I. Again these figures should be treated with some caution as advances in technology can be both rapid and dramatic. However at the current time they are an adequate guide to what may be achieved in reasonable production quantities without too much difficulty. Clearly the cost of an additional resistor or two is fairly small and so the inclusion of extra resistors to ease adjustment and reduce structural sensitivity may well be beneficial.

The equipment used to adjust hybrid circuits is nowadays almost invariably an automatic laser trimmer. For passive trimming to achieve a certain resistance, the added value relative to the total cost of the filter is small and will be ignored. However when performing active adjustment it is necessary to trim at a very much slower rate so that any transient disturbances to the circuit as a result of the laser light or change in resistance value have had time to decay to an insignificant level. In addition the equipment necessary for active trimming is considerably more complex, and hence expensive, than that required for passive trimming. The added value of active trimming can therefore be significant and is thus one of the design parameters to be taken into account.

In summary, the design of the filter should require a minimum number of capacitors and amplifiers and the capacitors should ideally be $1 \mathrm{nF}$ or less and of $10 \%$ tolerance. For a filter in the frequency range up to about $4 \mathrm{kHz}$, capacitors of this value imply that the resistors need to be in the approximate range $10 \mathrm{k} \Omega$ to $100 \mathrm{k} \Omega$ (in some designs the range is wider than this). To take up the capacitor tolerance and to allow for initial manufacturing spread some of the resistors will need to be trimmed by $30 \%$ to $50 \%$. This is probably most readily achieved using thick film technology and the design therefore needs to be able to use passively trimmed resistors with a $0.5 \%$ to $1 \%$ tolerance, 50 to $100 \mathrm{ppm}$ temperature coefficient, 5 to $10 \mathrm{ppm}$ differential temperature coefficient, $0.2 \%$ to $0.4 \%$ life stability and to be such that active trimming of certain resistors to within $0.5 \%$ to $1 \%$ of its final (but unknown) value will not result in unduly high sensitivity of the filter characteristics.

\section{FILTER STRUCTURES}

Almost universally active filters are designed via consideration of the pole and zero positions of a mathematical function that approximates the ideal characteristics required. It is possible to divide these functions into two broad classifications, (a) those derived from an ideal low pass characteristic and (b) those whose pole zero locations must be optimised 


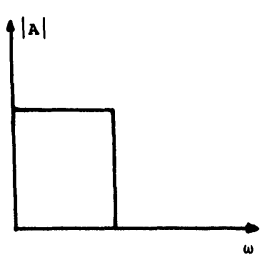

(a) Lowpass

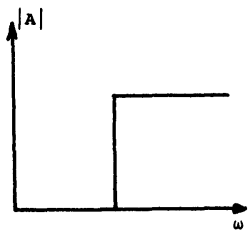

(b) Highpass

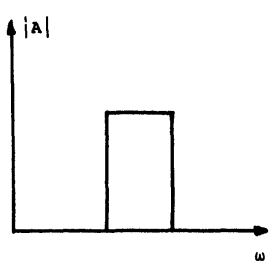

(c) Bandpass

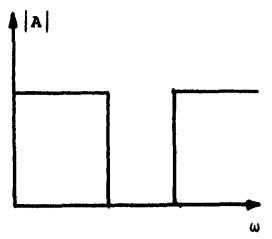

(d) Bandstop

FIGURE 3 Ideal lowpass, bandpass, bandstop and highpass filter functions.

using numerical techniques. In category (a) are the ideal lowpass, highpass, bandpass and bandstop filters as shown in Figure 3 (the latter three being obtained through transforming the lowpass function). These ideal characteristics may be approached arbitrarily closely by increasing the complexity or order of the filter function, (and consequently the complexity and cost of the final circuit). In general these functions can be derived using analytic techniques and an expression for the exact pole and zero positions evaluated. In case of category (b) functions, of which an example is shown in Figure 4, it is necessary to use numerical techniques to find the approximate pole and zero positions that will produce the necessary transfer function.

However as far as the active filter circuit is concerned it is only necessary to specify the particular pole and zero positions required (derived using either

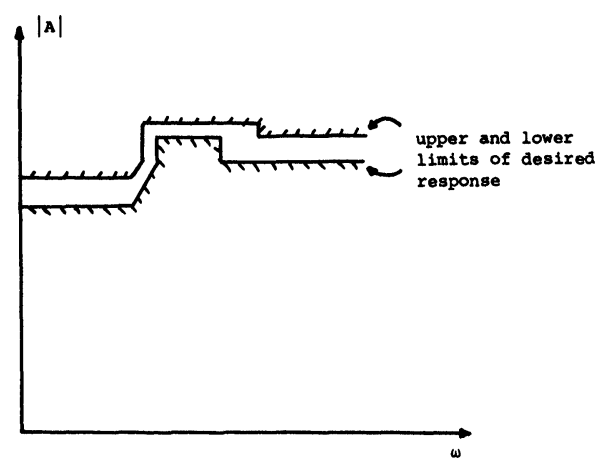

FIGURE 4 Typical filter function that requires numerical optimisation.

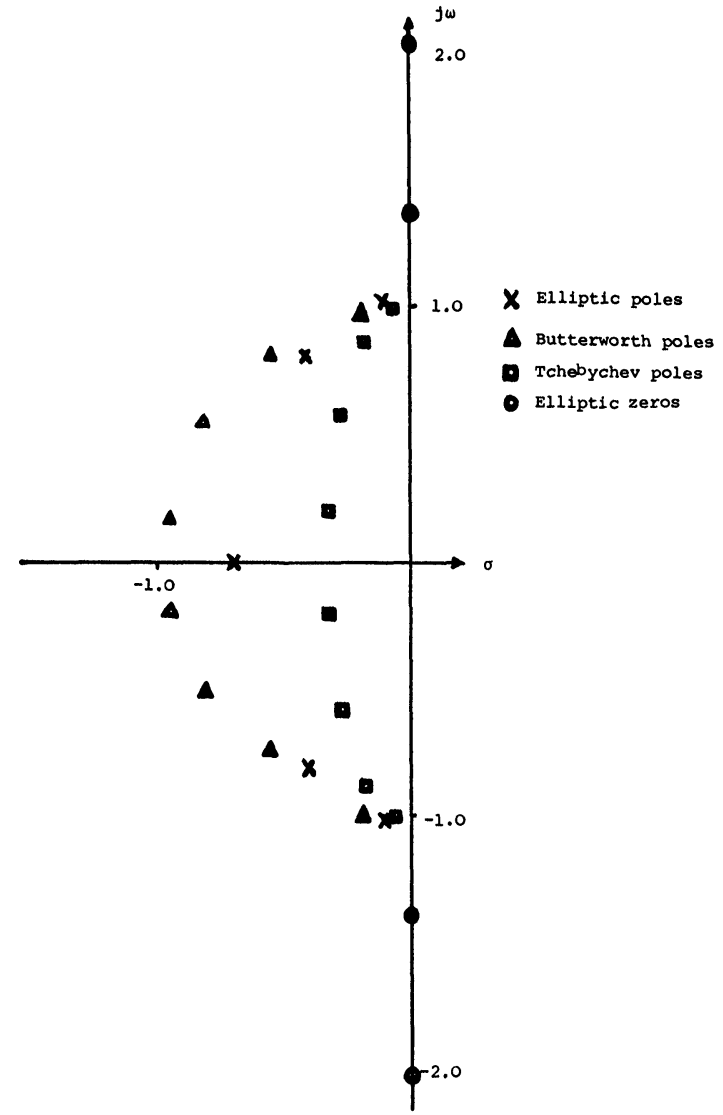

FIGURE 5 Pole positions for three representative filters.

analytic or numeric techniques), and hence, in general, any filter characteristics may be obtained.

Figure 5 shows the pole zero positions of three typical lowpass filter functions, an 8 pole Butterworth, an 8 pole $0.1 \mathrm{~dB}$ ripple Tchebychev and a

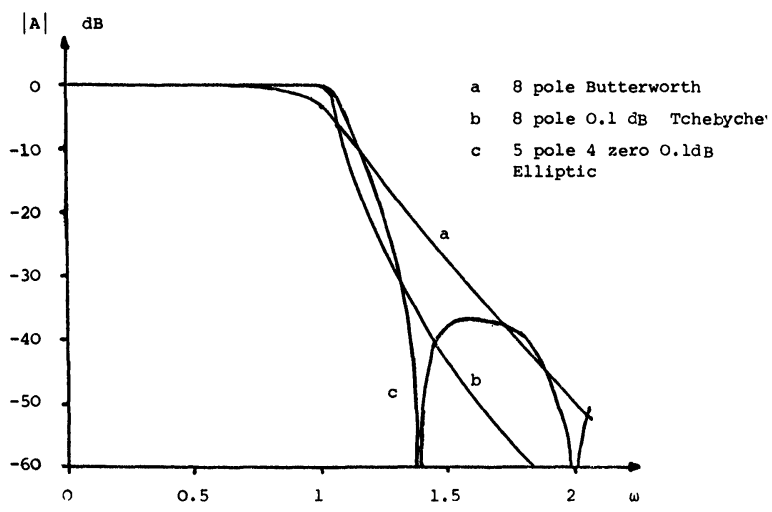

FIGURE 6 Transfer Characteristics for filters defined in Figure 6a. 
TABLE II

Pole positions for the filters of Figure 6a transferred to bandpass filters.

\begin{tabular}{|c|c|c|c|c|c|c|}
\hline & \multicolumn{3}{|c|}{ Prototype lowpass } & \multicolumn{3}{|c|}{ Bandpass } \\
\hline & \multicolumn{2}{|c|}{ Pole positions } & \multirow{2}{*}{$\frac{\text { Zero positions }}{\omega_{\infty}}$} & \multicolumn{2}{|c|}{ Pole positions } & \multirow{2}{*}{$\frac{\text { Zero positions }}{\omega_{\infty}}$} \\
\hline & $\omega_{r}$ & $Q$ & & $\omega_{r}$ & $Q$ & \\
\hline $\begin{array}{l}\text { Five pole four } \\
\text { zero elliptic }\end{array}$ & $\begin{array}{l}0.9099 \\
1.0658 \\
0.6971\end{array}$ & $\begin{array}{r}1.1197 \\
5.3938 \\
\text { eal pole) }\end{array}$ & $\begin{array}{l}1.3916 \\
2.0299\end{array}$ & $\begin{array}{l}0.9950 \\
0.9168 \\
1.0798 \\
0.8945 \\
1.1068\end{array}$ & $\begin{array}{r}7.1362 \\
12.2853 \\
12.2853 \\
50.6395 \\
50.6395\end{array}$ & $\begin{array}{l}1.1438 \\
0.8655 \\
1.2185 \\
0.8125 \\
1 \text { zero at } 0.0 \\
1 \text { zero at } s \rightarrow \infty\end{array}$ \\
\hline $\begin{array}{l}\text { Eight pole } \\
\text { Tchebychev }\end{array}$ & $\begin{array}{l}0.3823 \\
0.6456 \\
0.8941 \\
1.0344\end{array}$ & $\begin{array}{l}0.5928 \\
1.1809 \\
2.4478 \\
8.0648\end{array}$ & & $\begin{array}{l}0.9747 \\
1.0158 \\
0.9382 \\
1.0552 \\
0.9113 \\
1.0864 \\
0.8971 \\
1.10356\end{array}$ & $\begin{array}{l}15.4333 \\
15.4333 \\
18.2327 \\
18.2327 \\
27.3456 \\
27.3456 \\
77.9909 \\
77.9909\end{array}$ & $\begin{array}{l}8 \text { zeros at } 0.0 \\
8 \text { zeros at } s \rightarrow \infty\end{array}$ \\
\hline $\begin{array}{l}\text { Eight pole } \\
\text { Butterworth }\end{array}$ & $\begin{array}{l}1.0000 \\
1.0000 \\
1.0000 \\
1.0000\end{array}$ & $\begin{array}{l}0.5098 \\
0.6013 \\
0.9000 \\
2.5629\end{array}$ & & $\begin{array}{l}0.9756 \\
1.0148 \\
0.9408 \\
1.0523 \\
0.9152 \\
1.0817 \\
0.9017 \\
1.0979\end{array}$ & $\begin{array}{r}5.0734 \\
5.0734 \\
5.9927 \\
5.9926 \\
8.9860 \\
8.9860 \\
25.6244 \\
25.6244\end{array}$ & $\begin{array}{l}8 \text { zeros at } 0.0 \\
8 \text { zeros at } s \rightarrow \infty\end{array}$ \\
\hline
\end{tabular}

5 pole $0.1 \mathrm{~dB}$ ripple, $-35 \mathrm{~dB}$ stopband attenuation elliptic filter, while Figure 6 shows a comparison of the transfer functions. Table II shows the pole-zero positions of these functions transformed into a bandpass function with a $20 \%$ bandwidth using the standard geometric transform.

It can be seen that certain functions have faster rates of cut off than others for a given number of poles. The elliptic function gives the fastest initial rate of cut off, but the response does not decay as fast as the Tchebychev function further into the stopband. In comparison the Butterworth function does not seem to perform very well. As will be seen shortly however it is an easier filter to make.

It is also worth pointing out that other parameters are often specified, of which the most important is probably group delay variation. Each of these filter types and each order of filter will have a different group delay characteristic. Interestingly the inclusion of zeros on the $j \omega$ axis in the transmission function does not alter the group delay variation in the passband of the filter and therefore the group delay is controlled in the passband by the pole positions alone. The general transfer function for filters is

$$
T(s)=H \cdot \frac{\sum_{i=0}^{m} a_{i} s^{i}}{\sum_{j=0}^{n} b_{j}, s^{j}} \text { where } m \leqslant n
$$

To relate this to the pole and zero positions it is necessary to factorise this function

$$
T(s)=H \cdot \frac{N(s)}{\prod_{i=1}^{k}\left(s^{2}+\frac{\omega_{r i}}{Q} s+\omega_{r i}^{2}\right) \prod_{j=2 k+1}^{n}\left(s+\omega_{j}\right)}
$$

where $2 k$ is the number of poles that occur in complex conjugate pairs and $(n-2 k)$ is the number of real poles at frequencies $\omega_{j} . Q$ and $\omega_{r}$ are the parameters used to describe the pole positions.

Each type of filter will have a different form of 


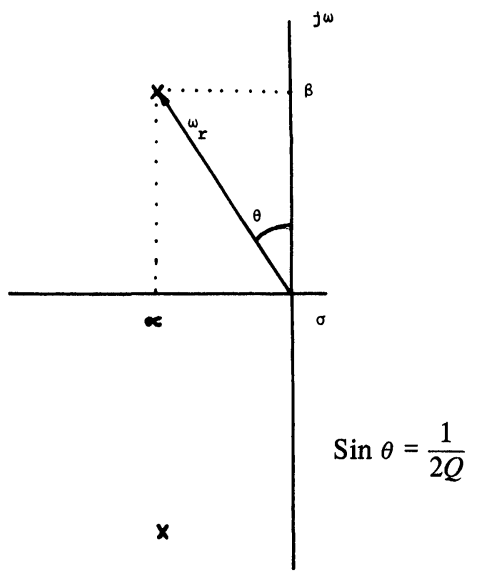

FIGURE 7 Definition of $Q$ and $\omega_{r}$ from the pole position.

$N(s)$. For a lowpass function without transmission zeros

$$
N(s)=1
$$

For a lowpass function with transmission zeros

$$
N(s)=\prod_{p=1}^{r}\left(s^{2}+\omega_{o p}^{2}\right) \quad r \leqslant \frac{n}{2}
$$

For a bandpass function with zeros only at $s=0+0_{j}$ and $s \rightarrow \infty, N(s)$ is of the form

$$
N(s)=s^{r}
$$

For a bandpass function with transmission zeros on the $j \omega$ axis

$$
N(s)=s^{r} \prod_{p=r+1}^{u}\left(s^{2}+\omega_{a p}^{2}\right) \prod_{q=1}^{v}\left(s^{2}+\omega_{b q}^{2}\right)
$$

where $\omega_{a}$ are the transmission zeros below the passband frequencies and $\omega_{b}$ are those above it. For analytic functions derived by applying the standard geometric transformation to a lowpass function then there will be equal numbers of $\omega_{a}$ and $\omega_{b}$. However it is not necessary that this shall be so and examples of numerically derived functions where this is not so are given by Saraga, Haigh and Barker. ${ }^{19,20}$

It is possible to relate the pole positions of these functions to the $Q$ and $\omega_{r}$ values as shown in Figure 7, and it is also possible to relate the sensitivity of the pole positions to the transfer response of an individual stage.

$$
T^{1}(s)=\frac{1}{s^{2}+\frac{\omega_{r}}{Q} s+\omega_{r}^{2}}
$$

In general sensitivity is defined as

$$
S_{x}^{y}=\frac{x}{y} \frac{\mathrm{d} y}{\mathrm{~d} x}
$$

where $y$ is a response and $x$ is a variable.

It can be shown that ${ }^{6}$

$$
S_{x}^{\left|T\left(s=j \omega_{r}\right)\right|}=\mid S_{x}^{Q}-S_{x}^{\omega_{r}}
$$

In general $S_{x}^{Q}$ and $S_{x}^{\omega} r$ can be reduced to quite low values $\left(\sim \frac{1}{2}\right)$. However at a frequency $\omega=\omega_{1}$ such that $\left|T\left(s=j \omega_{1}\right)\right|$ is $-3 \mathrm{~dB}$ of $\left|T\left(s=j \omega_{r}\right)\right|$ then

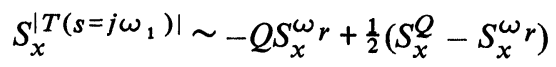

Therefore structurally, filter functions with a high $Q$ require components with greater stability and closer tolerance. It can be seen from Table II that in general the $Q$ factors for bandpass filters will in fact be higher than the lowpass designs from which they were derived.

There are basically two types of implementation of active filters in common use. The one first pointed out by Sallen and Key models the form of Eq. (2) exactly by applying second order feedback to an amplifier. The feedback also results in a low output impedance and it is therefore possible to cascade the sections resulting in the multiplication of the individual transfer functions each of which is of the general form

$$
T^{I}(s)=H^{I} \frac{1}{s^{2}+\frac{\omega_{r}}{Q} s+\omega_{r}^{2}}
$$

or

$$
T^{I}(s)=H^{I} \frac{s^{2}+\omega_{0}^{2}}{s^{2}+\frac{\omega_{r}}{Q} s+\omega_{r}^{2}}
$$

or

$$
T^{I}(s)=H^{I} \frac{s}{s^{2}+\frac{\omega_{r}}{Q} s+\omega_{r}^{2}}
$$

and hence can place one pair of poles and up to one pair of zeros. Alternatively, it is possible to realize the filter using the structure of an LC filter and to replace the undesirable components with a combination of resistors, capacitors and amplifiers. The advantage of doing this was first demonstrated by $\operatorname{Orchard}^{3}$ who pointed out that in the passband the insertion loss of such filters is zero $\mathrm{dB}$ and thus 
any deviation from the ideal components can therefore only increase the insertion loss. Hence at the ideal values the sensitivity of the filter function to changes in component values is zero for all components.

\section{TRIMMING}

The ability to trim the resistors of a hybrid module to compensate for variations in capacitor values using high speed automatic equipment is one of the most important advantages of the hybrid technique. There are basically two approaches to trimming. In the first the capacitors are measured and the resistor values calculated and then trimmed to this value. For instance in the case of the Sallen and Key lowpass filter section the transmission function is

$$
T(s)=\frac{K \frac{G_{1} G_{2}}{C_{3} C_{4}}}{s^{2}+\left[\frac{G_{1}+G_{2}}{C_{3}}+\frac{G_{2}(1-K)}{C_{4}}\right] s+\frac{G_{1} G_{2}}{C_{3} C_{4}}}
$$

where $G_{1}, G_{2}$ are the conductances that may be adjusted. $K$ is the gain of the amplifier and is of the form

$$
1+\frac{G_{3}}{G_{4}}
$$

and $C_{3}, C_{4}$ are the capacitors whose values are measured. The general form of the lowpass filter section is

$$
T(s)=\frac{H \omega_{r}^{2}}{s^{2}+\frac{\omega_{r}}{Q} s+\omega_{r}^{2}}
$$

By identifying the coefficients of Eq. (10) with those of Eq. (9) it can be seen that

$$
\begin{aligned}
& \text { d.c. gain }=H=K \\
& \omega_{r}=\sqrt{\frac{G_{1} G_{2}}{C_{3} C_{4}}} \\
& Q=\frac{1}{\sqrt{\frac{C_{4}}{C_{3}}}\left(\sqrt{\frac{G_{1}}{G_{2}}}+\sqrt{\frac{G_{2}}{G_{1}}}\right)+\sqrt{\frac{C_{3} G_{2}}{C_{4} G_{1}}}(1-K)}
\end{aligned}
$$

However, we are not interested in the relationships as given in Eqs (11), (12), (13) but in the values of the components necessary to obtain simultaneously the required $Q, \omega_{r}$ and d.c. gain, given the measured values of $C_{3}$ and $C_{4}$. In other words

$$
\begin{aligned}
& K=H \\
& G_{1}=\frac{\omega_{r}}{2}\left(\frac{C_{3}}{Q} \pm \sqrt{\frac{C_{3}^{2}}{Q^{2}}-4\left(C_{3} C_{4}+C_{3}^{2}(1-H)\right)}\right) \\
& G_{2}=\frac{C_{3} C_{4} \omega_{r}^{2}}{G_{1}}
\end{aligned}
$$

In practice the value of d.c. gain is often not of great importance and thus $K$ could be used as a trimming variable.

In the second approach circuit parameters are measured. These are related to the design parameters and changes in component values are calculated based upon the known relationship between the parameters and component values. For instance it may be possible in some particular circuit to write

$$
\left[\begin{array}{l}
\Delta \omega_{r} \\
\Delta Q \\
\Delta K
\end{array}\right]=\left[\begin{array}{lll}
\alpha_{11} & \alpha_{12} & \alpha_{13} \\
\alpha_{21} & \alpha_{22} & \alpha_{23} \\
\alpha_{31} & \alpha_{32} & \alpha_{33}
\end{array}\right]\left[\begin{array}{l}
\Delta R_{1} \\
\Delta R_{2} \\
\Delta R_{3}
\end{array}\right]
$$

when the components have their ideal values. Then, assuming that the errors in the design parameters are not too great, it is possible to calculate the changes in resistors necessary to correct for these errors. It is probably wise to alter these values by only a fraction of the predicted amount, and then to repeat the process until the errors in the measured values of $Q$, $\omega_{r}$ and d.c. gain are arbitrarily small.

A variation of this is possible if the matrix can be arranged to be of the form

$$
\left[\begin{array}{l}
\Delta P_{1} \\
\Delta P_{2} \\
\Delta P_{3}
\end{array}\right]=\left[\begin{array}{ccc}
\alpha_{11} & 0 & 0 \\
\alpha_{21} & \alpha_{22} & 0 \\
\alpha_{31} & \alpha_{32} & \alpha_{33}
\end{array}\right]\left[\begin{array}{l}
\Delta R_{1} \\
\Delta R_{2} \\
\Delta R_{3}
\end{array}\right]
$$

where $[\Delta P]$ are the design parameters and $[\Delta R]$ are the resistor changes. In this situation parameter 1 can be set using resistor 1 . This will also affect parameters 2 and 3. Parameter 2 can then be set using resistor 2; this will also affect parameter 3 , but not parameter 1 . Finally, parameter 3 can be set using resistor 3 . In this way relatively few adjustments are required.

The design parameters are defined by

$$
T(s)=\frac{H \omega_{r}^{2}}{s^{2}+\frac{\omega_{r}}{Q} s+\omega_{r}^{2}}
$$




$$
\begin{aligned}
& T\left(s-j \omega_{r}\right)=j H Q \\
& T(s=0+j 0)=H
\end{aligned}
$$

Therefore it can be seen that the d.c. gain can be readily measured, $\omega_{r}$ is the frequency at which the phase shift is $-90^{\circ}$ and the $Q$ is given by the ratio of the gain at $s=j \omega_{r}$ to that at d.c.

The predictive method has the virtue of conceptual simplicity and does not require very much in the way of instrumentation connected to the trimmer. There are however some potential problems of logistics in measuring the capacitor values. If this is done prior to assembly then it is necessary to ensure that the components are correctly assembled. If it is done after assembly then the effects of the parallel components need to be eliminated. Further this technique does not allow for "stray" components. The iterative method requires a relatively large amount of measurement and computation and can therefore be time consuming. The method using a triangular matrix is probably the most effective since it requires a minimum of trimming and no inter-trim computation. If the filter function is relatively simple then it may prove possible to sort the capacitors into bands, say $2 \%$ wide, and to trim the resistors in batches accordingly.

\section{SALLEN AND KEY CIRCUIT}

In their original paper Sallen and Key published several circuits that could be used as various second order stages, but it is the lowpass circuit shown in Figure 8 that is the one usually credited to them. It is

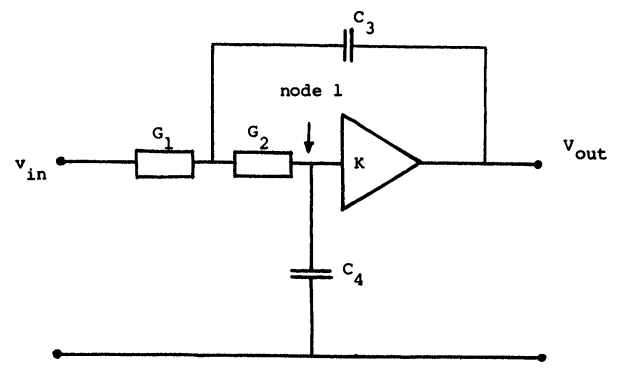

FIGURE 8 Sallen and Key lowpass circuit.

also a simple yet very interesting example of the dilemma faced by the engineer attempting to manufacture an active filter. The transfer function for this circuit is

$$
T(s)=\frac{K \frac{G_{1} G_{2}}{C_{3} C_{4}}}{s^{2}+s\left[\frac{\left(G_{1}+G_{2}\right)}{C_{3}}+\frac{G_{2}(1-K)}{C_{4}}\right]+\frac{G_{1} G_{2}}{C_{3} C_{4}}}
$$

comparing this to the desired transfer function

$$
\begin{aligned}
& \text { d.c. gain }=K \\
& \omega_{r}=\sqrt{\frac{G_{1} G_{2}}{C_{3} C_{4}}} \\
& Q=\frac{1}{\sqrt{\frac{C_{4}}{C_{3}}}\left(\sqrt{\left.\frac{G_{1}}{G_{2}}+\sqrt{\frac{G_{2}}{G_{1}}}\right)+\sqrt{\frac{C_{3}}{C_{4}} \frac{G_{2}}{G_{1}}}(1-K]}\right.}
\end{aligned}
$$

Let us now consider two distinct conditions that might be beneficial.

Firstly let the gain $K$ be unity. This configuration requires no feedback resistors and gives rise to a very stable value of $K$ over a wide bandwidth. It turns out, however, that when $K=1$ the circuit is sensitive to changes in the open loop gain. This will be discussed further in the section on amplifier limitation. Clearly the d.c. gain is fixed at unity. $Q$ is then given by,

$$
Q=\frac{\sqrt{\frac{C_{3}}{C_{4}}}}{\sqrt{\frac{G_{1}}{G_{2}}}+\sqrt{\frac{G_{2}}{G_{1}}}}
$$

This gives a minimum ratio of $C_{3} / C_{4}$ as

$$
\left(\frac{C_{3}}{C_{4}}\right)_{\min }=4 Q^{2} \quad \text { when } G_{1}=G_{2}
$$

If the lowest value of $C_{4}$ acceptable to dominate the strays is $\sim 100 \mathrm{pF}$ and the upper "economic" value is $2 \mathrm{nF}$ (see Figure 1), then $Q_{\max } \sim \sqrt{ } 5$, a very low value. Also it is not possible to set up the required triangular matrix for trimming and so simple functional adjustment is not possible.

The sensitivity values are given by

$$
\begin{aligned}
& S_{G_{1}}^{\omega_{r}}=S_{G_{2}}^{\omega_{r}}=-S_{C_{3}}^{\omega_{r}}=-S_{C_{4}}^{\omega_{r}}=\frac{1}{2} \\
& S_{G_{1}}^{Q}=S_{G_{2}}^{Q}=0 \text { (at the "ideal" values) } \\
& S_{C_{3}}^{Q}=-S_{C_{4}}^{Q}=\frac{1}{2}
\end{aligned}
$$


These values are low.

The second condition that will be examined is that of equal capacitors. $Q$ is now given by

$$
Q=\frac{1}{\sqrt{\frac{G_{1}}{G_{2}}+\sqrt{\frac{G_{2}}{G_{1}}}(2-K)}}
$$

It can be seen that for any value of $G_{1} / G_{2}$ it is possible to increase $Q$ indefinitely until it becomes infinite by increasing $K$ and, if the value of d.c. gain is not important, then $K$ may be used as an adjustment variable. Since $\omega_{r}$ is not a function of $K$, then a triangular adjustment matrix may be set up and hence simple non-iterative trimming is possible. The only problem with the adjustment is the necessity to measure repeatedly the value of d.c. gain, since this will alter as $K$ is adjusted. This can be overcome by measuring the transfer function at node 1 on Figure 8, for which the transfer function is

$$
\frac{V_{1}}{V_{\text {in }}}=\frac{\frac{G_{1} G_{2}}{C_{3} C_{4}}}{s^{2}+s\left[\frac{G_{1}+G_{2}}{C_{3}}+\frac{G_{2}(1-K)}{C_{4}}\right]+\frac{G_{1} G_{2}}{C_{3} C_{4}}}
$$

and hence the d.c. gain is always 1 . The adjustment procedure is therefore to set the input frequency to $\omega_{r}$ and to increase $G_{1}$ or $G_{2}$ until the phase shift is $-90^{\circ}$ and then to adjust $K$ until the gain at the frequency is $Q$. The problem of the variable d.c. gain of the filter can be overcome by adding an extra amplifier or attenuator which is adjusted last of all. The drawback in using this approach can be seen from considering the sensitivity function

$$
S_{K}^{Q}=Q\left(2 \sqrt{\frac{G_{2}}{G_{1}}}+\sqrt{\frac{G_{1}}{G_{2}}}\right)-1
$$

This has a minimum value of

$$
S_{K_{\min }}^{Q}=2 \sqrt{ } 2 Q-1
$$

when

$$
G_{1}=2 G_{2}
$$

$Q$ is then given by

$$
Q=\frac{1}{2 \sqrt{ } 2-\frac{1}{\sqrt{ } 2} K}=\frac{\sqrt{ } 2}{4-K}
$$

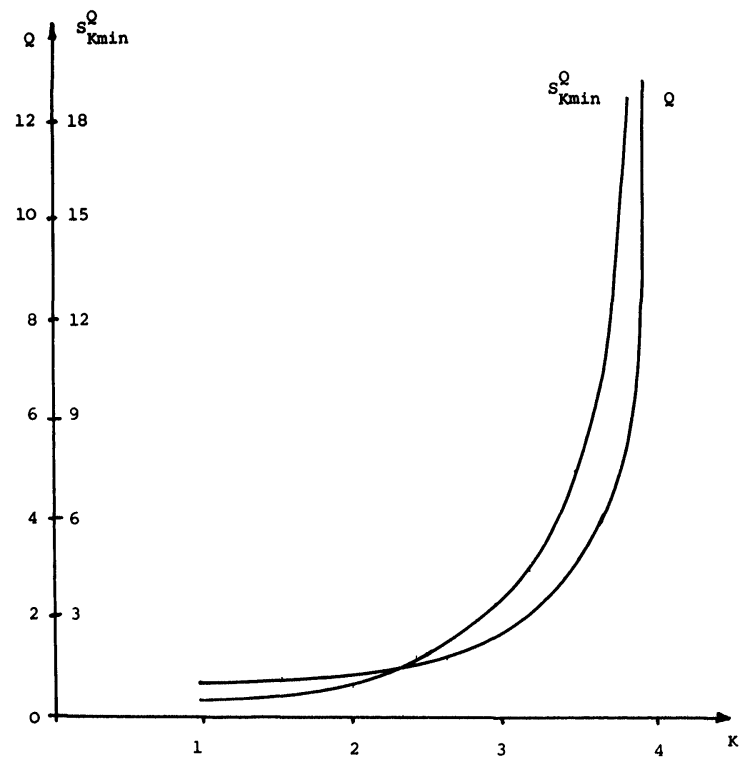
FIGURE $9 \quad Q$ vs $K$ and $S_{K_{\min }}^{Q}$ vs $K$ for equicapacitor
Sallen and Key circuit.

The relationship between $Q$ and $K$ for Eq. (35) is given in Figure 9. In order to examine the effect of this value of $S_{K}^{Q}$, consider a filter with a $Q$ of 5 and a technology in which $K$ may be adjusted to $\pm 0.25 \%$. $S_{K}^{Q}$ is then 13.1 and the consequent uncertainty in $Q$ is 3.3\% which implies an approximate uncertainty in the transmission function of $0.28 \mathrm{~dB}$ around the frequency $\omega=\omega_{r}$. For several stages errors will be accumulative and whilst they may not all be as large as this for a given adjustment accuracy, it is likely that the total error will be unacceptably large. The sensitivities with respect to the non-adjusted components are at the nominal values.

$$
\begin{aligned}
& S_{G_{1}}^{Q}=-S_{G_{2}}^{Q}=-\sqrt{ } 2 Q+\frac{1}{2} \\
& S_{C_{3}}^{Q}=-S_{C_{4}}^{Q}=\frac{5 Q}{2 \sqrt{ } 2}-\frac{1}{2} \\
& S_{G_{1}}^{\omega_{r}}=S_{G_{2}}^{\omega_{r}}=-S_{C_{3}}^{\omega_{r}}=-S_{C_{4}}^{\omega_{r}}=\frac{1}{2}
\end{aligned}
$$

These values can also be seen to be fairly high.

It is possible to reduce the component and gain sensitivities by increasing the capacitor spread; however reasonably high sensitivities to passive components and closed loop gain $K$ will always be a feature of this design. 


\section{RAUCH CIRCUIT}

This circuit, the lowpass version of which is shown in Figure 10, relies on the amplifier having a large gain and as the dominant pole for commonly used amplifiers is only a few hertz, it is to be expected that the performance of this type of circuit will not be as good as the Sallen and Key circuit, which requires a gain near unity. The transfer function for this circuit is

$$
T(s)=\frac{-\frac{G_{1} G_{2}}{C_{4} C_{5}}}{s^{2}+\frac{G_{1}+G_{2}+G_{3}}{C_{4}} s+\frac{G_{2} G_{3}}{C_{4} C_{5}}}
$$

for which d.c. gain $=-\frac{G_{1}}{G_{3}}$

$$
\begin{aligned}
\omega_{r} & =\sqrt{\frac{G_{2} G_{3}}{C_{4} C_{5}}} \\
Q & =\sqrt{\frac{C_{4}}{C_{5}}} \frac{1}{\frac{G_{1}}{\sqrt{G_{2} G_{3}}}+\sqrt{\frac{G_{2}}{G_{3}}}+\sqrt{\frac{G_{3}}{G_{2}}}}
\end{aligned}
$$

Clearly the expression for $Q$ imposes a well defined minimum ratio of $C_{4} / C_{5}$ at $4 Q^{2}$ as $G_{1} \rightarrow 0$. However this implies that the d.c. gain tends to zero. Therefore choosing the d.c. gain to be -1 gives $C_{4} / C_{5 \text { min }}$ as $8 Q^{2}$ when $G_{2}=2 G_{3}$. Simple non-iterative tuning of this circuit is not possible. However if the d.c. gain is not of importance, then $\omega_{r}$ and $Q$ may be trimmed independently by first of all tuning either $G_{2}$ or $G_{3}$ to set $\omega_{r}$ and then adjusting $G_{1}$ to set the $Q$.

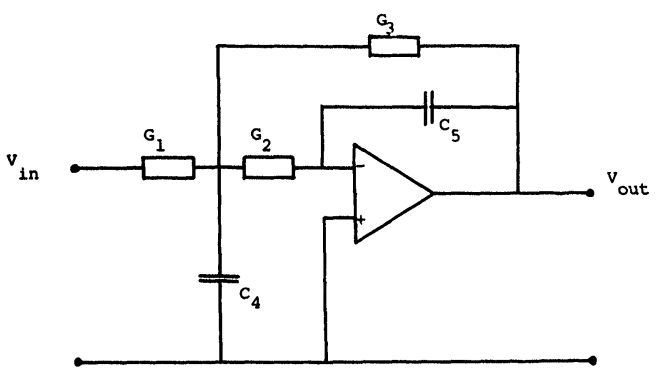

FIGURE 10 Rauch lowpass circuit.

\section{STATE VARIABLE CIRCUIT}

The state variable circuit, shown in Figure 11, is essentially a double integrator and a summing

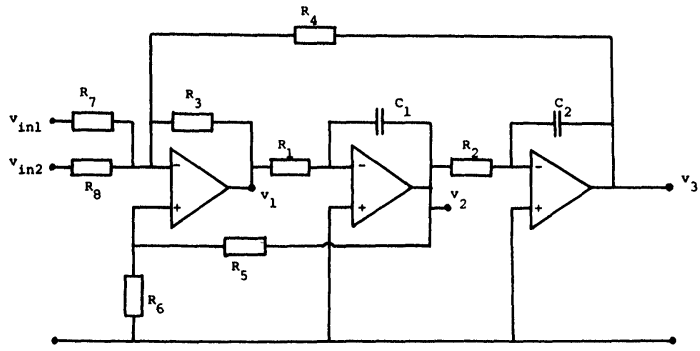

FIGURE 11 State variable circuit.

amplifier in a feedback loop. It is sometimes known as the universal filter section or "ring of three". The circuit has two independent inputs $v_{\text {in } 1}$ and $v_{\text {in } 2}$ and three outputs $v_{1}, v_{2}$, and $v_{3}$ and is capable of forming all the common filter functions. For the lowpass circuit, with or without a transmission zero, the relevant transmission functions are

$$
\begin{gathered}
v_{1}=\frac{-s^{2}\left(\frac{R_{3}}{R_{7}} v_{\mathrm{in} 1}+\frac{R_{3}}{R_{8}} v_{\mathrm{in} 2}\right)}{s^{2}+\frac{s}{R_{1} C_{1}} \frac{R_{3} R_{6}}{R_{5}+R_{6}}\left(\frac{1}{R_{3}}+\frac{1}{R_{4}}+\frac{1}{R_{7}}+\frac{1}{R_{8}}\right)+\frac{R_{3}}{R_{4}} \frac{1}{R_{1} R_{2}}} \\
v_{3}=\frac{-\frac{1}{s^{2}+\frac{s}{R_{1} C_{1}} \frac{R_{1} R_{2} C_{1} C_{2}}{R_{5}+R_{6}}\left(\frac{1}{R_{3}}+\frac{1}{R_{7}} v_{\mathrm{in} 1}+\frac{R_{3}}{R_{8}} v_{\mathrm{in} 2}\right)}}{\left.R_{7}+\frac{1}{R_{8}}\right)+\frac{R_{3}}{R_{4}} \frac{1}{R_{1} R_{2} C_{1}}}
\end{gathered}
$$

The operation of this circuit is that $v_{1}$ and $v_{3}$ form the inputs $v_{\mathrm{in} 1}$ and $v_{\mathrm{in} 2}$ to the following stage. Since these two inputs are weighted and summed by preset amounts to both outputs, a transmission zero may be formed. Alternatively only the $v_{3}$ output may be used and a lowpass circuit with no zeros at $s<\infty$ formed. For this circuit,

$$
\begin{gathered}
\omega_{r}=\sqrt{\frac{R_{3}}{R_{4}} \frac{1}{R_{1} R_{2} C_{1} C_{2}}} \\
Q=\frac{\left(1+\frac{R_{5}}{R_{6}}\right) \sqrt{\frac{R_{1} C_{1}}{R_{2} C_{2}}}}{\sqrt{\frac{R_{4}}{R_{3}}+\sqrt{\frac{R_{3}}{R_{4}}}+\sqrt{R_{3} R_{4}}\left(\frac{1}{R_{7}}+\frac{1}{R_{8}}\right)}}
\end{gathered}
$$

and for the lowpass version

$$
\text { d.c. gain; } \frac{v_{3}}{v_{1}}=-\frac{R_{4}}{R_{7}} \quad \frac{v_{3}}{v_{2}}=-\frac{R_{4}}{R_{8}}
$$


It can be seen therefore that as $Q$ may be set arbitrarily large by suitable choice of $R_{6}$ and equal valued capacitors may be used. Further it is possible to set up a triangular adjustment matrix and hence simple non-iterative trimming is possible.

\section{LIM CIRCUIT}

This circuit, based on the Twin T network, was originally discovered by $\mathrm{J}$. T. $\mathrm{Lim}^{7}$ and subsequently rediscovered by A. Sedra. ${ }^{8}$ Like the state variable circuit it is capable of forming all the common filter sections. The general form of the circuit is shown in Figure 12 and it can be seen to have three inputs and one output. The transmission functions relevant to the lowpass circuits are

$$
\begin{aligned}
& \frac{v_{0}}{v_{\mathrm{a}}}=\frac{\omega_{r}^{2}}{s^{2}+\frac{\omega_{r}}{Q} s+\omega_{r}^{2}} \\
& \frac{v_{0}}{v_{\mathrm{b}}}=\frac{s^{2}}{s^{2}+\frac{\omega_{r}}{Q} s+\omega_{r}^{2}}
\end{aligned}
$$

These transmission functions assume that the input ports are driven from a zero impedance. $Q$ and $\omega_{r}$ will be defined later on.

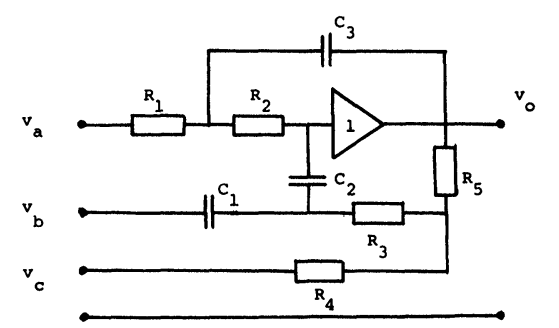

FIGURE 12 General form of Lim circuit.

It will be noticed that this circuit uses three capacitors and as such it is a third order circuit. However it was shown by Moschytz $z^{9}$ using the results of Hastings ${ }^{10}$ that when the Twin T is adjusted to give a perfect null, pole zero cancellation occurs and a biquadratic transfer function results. The basic Twin T circuit is shown in Figure 13 for which the transfer function at balance is

$$
T(s)=\frac{s^{2}+\omega_{n}^{2}}{s^{2}+\left(\omega_{n}^{2} R_{1} C_{3}+\frac{1}{R_{1} C_{3}}\right) s+\omega_{n}^{2}}
$$

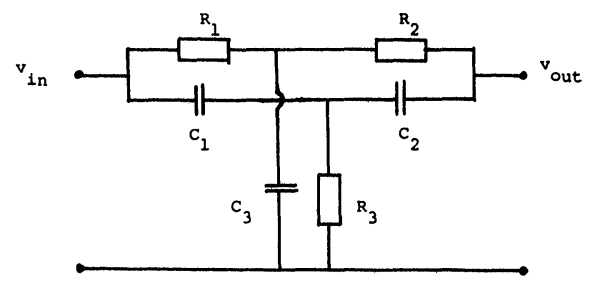

FIGURE 13 Twin T circuit.

and the balance condition is

$$
\left(C_{1}+C_{2}\right) R_{3}=\frac{R_{1} R_{2}}{R_{1}+R_{2}} C_{3}
$$

and $\omega_{n}$ is given by

$$
\omega_{n}^{2}=\frac{1}{C_{1} C_{2} R_{3}\left(R_{1}+R_{2}\right)}=\frac{C_{1}+C_{2}}{C_{1} C_{2} C_{3} R_{1} R_{2}}
$$

It will be observed that a balance may be achieved with any set of capacitors; in particular when equal valued capacitors are used the balance condition reduces to

$$
2 R_{3}=\frac{R_{1} R_{2}}{R_{1}+R_{2}}
$$

The basic structure of the Lim circuit, showing how it is developed from the Twin T network is given in Figure 14, for which the transfer function is

$$
T=\frac{T_{12}}{1-T_{42}-K T_{32}}
$$

where $T_{i j}$ is the voltage transfer function from node $i$ to node $j$. It may be shown that the poles of this function are the solution to

$$
s^{2}+\frac{1}{C_{1} R_{3}}(1-K) s+\frac{C_{1}+C_{2}}{C_{1} C_{2} C_{3} R_{1} R_{2}}=0
$$

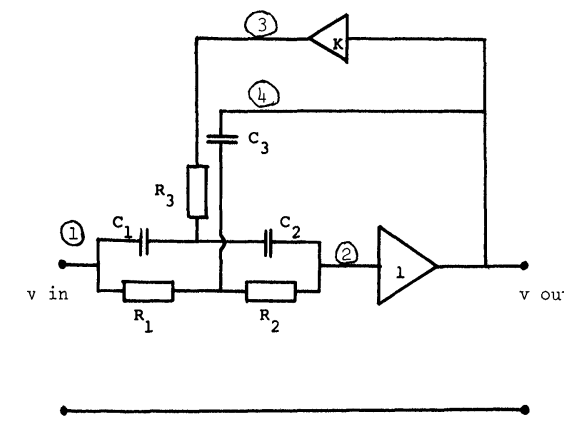

FIGURE 14 Derivation of Lim circuit from Twin T. 
when the Twin $\mathrm{T}$ is balanced. Thus

$$
\begin{gathered}
\omega_{r}=\sqrt{\frac{C_{1}+C_{2}}{C_{1} C_{2} C_{3} R_{1} R_{2}}} \\
Q=\sqrt{\frac{\left(C_{1}+C_{2}\right)}{\frac{C_{1} C_{2} C_{3} R_{1} R_{2}}{(1-K) / C_{1} R_{3}}}}
\end{gathered}
$$

Letting $C_{1}=C_{2}=C_{3}$

$$
\begin{aligned}
\omega_{r} & =\frac{1}{C} \sqrt{\frac{2}{R_{1} R_{2}}} \\
Q & =\frac{R_{3}}{1-K} \sqrt{\frac{2}{R_{1} R_{2}}}
\end{aligned}
$$

The values $R_{1}=R, R_{2}=2 R, R_{3}=R / 3$ are often used thus making

$$
\begin{aligned}
\omega_{r} & =\frac{1}{R C} \\
Q & =\frac{1}{3(1-K)}
\end{aligned}
$$

or

$$
K=1-\frac{1}{3 Q}
$$

The value of $K$ is therefore always positive and less than 1 for $Q>1 / 3$ and hence it may be realised by a potential divider circuit. In practice an additional resistor is included so that the adjustment of $Q$ and the balancing of the Twin $\mathrm{T}$ are independent. Thus the circuit of Figure 12 has been derived for which

$$
\begin{aligned}
& R_{4}=(3 Q-1) R_{5} \\
& \frac{R_{4} R_{5}}{R_{4}+R_{5}}+R_{3}=R / 3
\end{aligned}
$$

This spread in resistor values is reasonably low for a circuit with low sensitivity. If $R_{3} \gg R_{4} / R_{5}$, then adjusting $R_{4}$ does not unbalance Eq. (51) to any significant extent.

In order to form transmission zeros the signal is injected into both ports a and $c$ with a potential divider at the input to one or both ports. Thus

$$
T_{a \text { and } c}=\frac{\gamma s^{2}+\delta \omega_{r}^{2}}{s^{2}+\frac{\omega_{r}}{Q} s+\omega_{r}^{2}}
$$

This may be realised using the circuit of Figure 15

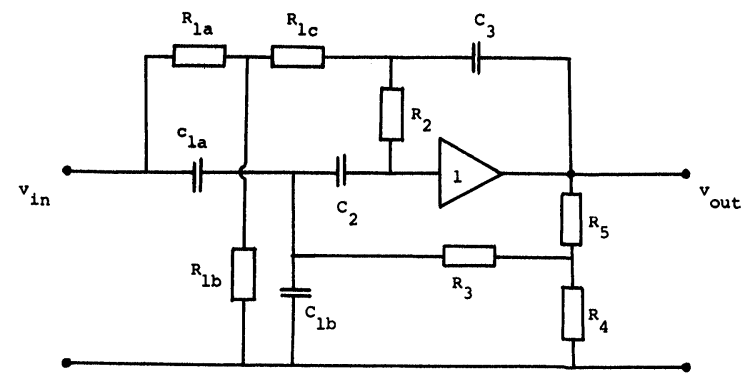

FIGURE 15 Lim circuit with transmission zeros.

for which

$$
\begin{aligned}
& \gamma=C_{1 a} /\left(C_{1 a}+C_{1 b}\right) \\
& \delta=R_{1 b} /\left(R_{1 a}+R_{1 b}\right)
\end{aligned}
$$

and hence $\omega_{\infty}$ is given by

$$
\omega_{\infty}^{2}=\omega_{r}^{2} \frac{R_{1 b}}{R_{1 a}+R_{1 b}}\left(1+\frac{C_{1 b}}{C_{1 a}}\right)
$$

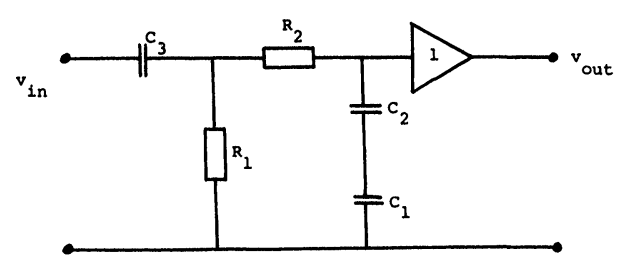

(a) Balance Btidged $\mathrm{T}$

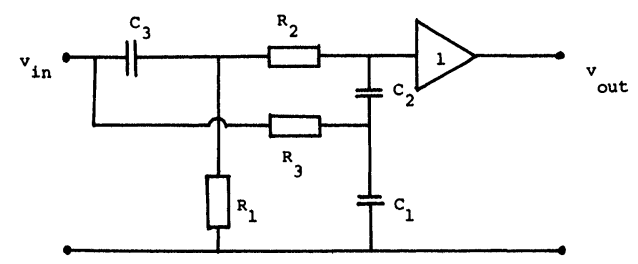

(b) Balance Twin I

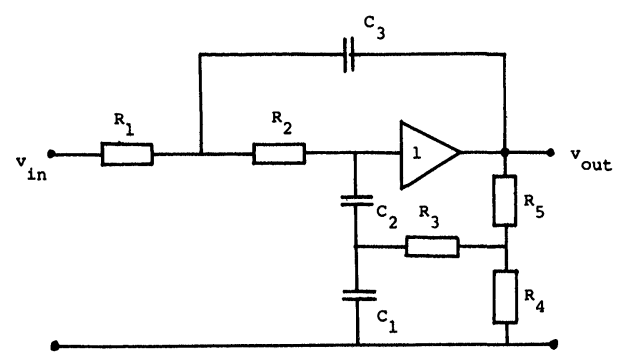

(c) Set $Q$

FIGURE 16 Adjustment subnetworks for Lim circuit. 
For $\omega_{\infty}<\omega_{r}, C_{1 b}$ may be omitted and the zero frequency set using $R_{1 a}$ and $R_{1 b}$. For $\omega_{\infty}>\omega_{r}$ (the case for lowpass circuits) $C_{1 a}$ and $C_{1 b}$ are necessary such that $C_{1 a}+C_{1 b}=C_{1} \cdot R_{1 a}$ and $R_{1 b}$ may be set to compensate for the tolerances of $C_{1 a}$ and $C_{1 b}$.

The major problem with the Lim circuit is balancing the Twin $\mathrm{T}$ since this requires the formation of subnetworks. The basis for this adjustment was first presented by Moschytz ${ }^{11}$ and modified for the Lim circuit $^{12}$ to use phase adjustment at a non-zero voltage rather than a voltage null adjustment. The circuits used for adjustment are shown in Figures $16 \mathrm{a}, 16 \mathrm{~b}$, and $16 \mathrm{c}$ where $C_{1}$ includes $C_{1 a}$ and $C_{1 b}$, $R_{1}$ includes $R_{1 a}, R_{1 b}$ and $R_{1 c}$ and $R_{3}$ includes $R_{3}$, $R_{4}$ and $R_{5}$.

The performance of the Lim circuit is very good, particularly with respect to amplifier variations. Its drawbacks are the necessity to use three or four capacitors and the subnetwork formation required for adjustment, which makes the manufacture of multistage circuits on a single substrate rather difficult.

\section{POSITIVE IMPEDANCE CONVERTER (P.I.C.)}

As stated earlier the LC ladder structure provides an inherently low sensitivity structure for filters; however simply replacing the inductors by circuits containing resistors, capacitors and amplifiers does not overcome one of the major problems, that of narrow capacitor tolerance in the capacitors of the original filter. However Bruton ${ }^{13}$ has pointed out that the voltage transfer function of a filter is not
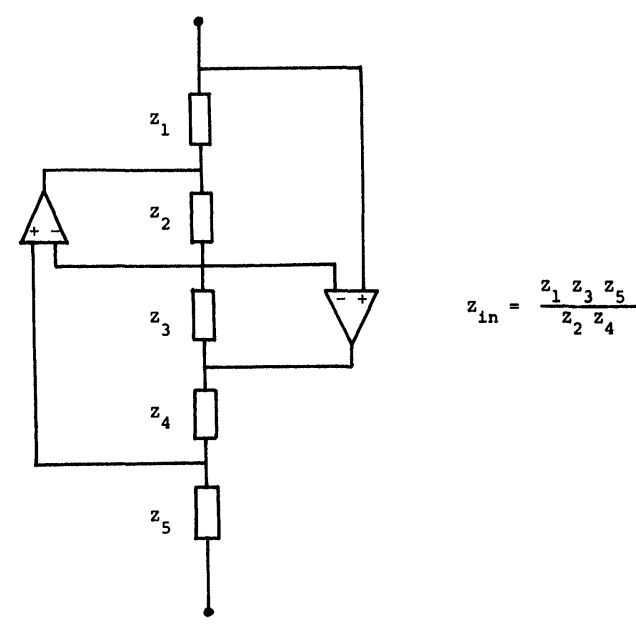

FIGURE 17 Antoniou's General Impedance Converter. altered by scaling all the components by some function of $s$. In particular it is possible to multiply all the impedances by $K / s$. In this way all the inductors of impedance sL become resistors of value $K L$ and the capacitors of impedance $1 / s C$ now have a value of $K / s^{2} C$. This latter impedance can be realised by using Antoniou's ${ }^{5,19}$ General Impedance Converter (GIC) shown in Figure 17. The GIC can, by proper choice of components, be made to have a very high $Q$ factor over a relatively wide frequency range and can be tuned to a specific impedance value by resistor adjustment alone and may use equal valued capacitors.

Two circuits using this technique are shown in Figure 18, the first simulates a five pole four zero elliptic filter and the second an 8 pole Tchebychev (or Butterworth) filter. In both, the "inductors" can be set simply by passive resistor adjustment. However in the second the "capacitors" cannot be adjusted "in situ". In the first circuit the zeros are set by the resonance of one of the general impedance converters with the resistor connected in series with it. It is possible therefore to tune the poles of this filter by placing the zeros correctly and further this can be done at the external terminals of the filter and for the whole filter. The tuning of each zero is independent; thus the trimming is not iterative.

While the capacitors in the general impedance converters may be of relatively wide tolerance, those that form the source and load impedance need to be

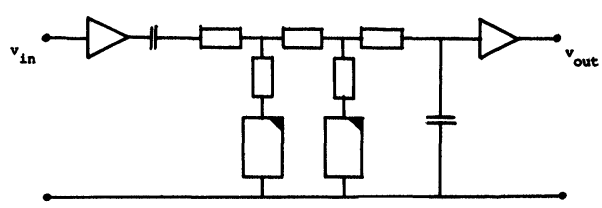

(a) Five pole four zoro Elliptic elrcult

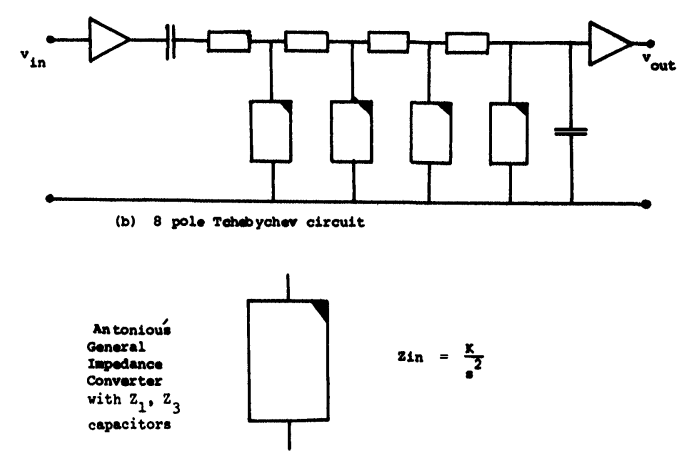

FIGURE 18 Two P.I.C. filter circuits. 
chosen with rather more discrimination. Most filter ${ }^{\dagger}$ component values for this type of filter are evaluated for equal valued source and load and so while the value of the source and load capacitors is not important, they do need matching to within a certain tolerance. The particular value of capacitance chosen is then used to calculate $K$.

Also to enable the amplifier to be biased properly the capacitors need to be shunted by high value resistors. This can mean an extra resistor print for just two resistors. This circuit gives rise to excellent performance but does require a larger number of amplifiers and capacitors than some other circuits; precisely the components that are most expensive! However the ease of adjustment and high performance often outweigh these considerations.

\section{OTHER CIRCUITS}

A great many circuits have been proposed for use as active filter sections. An exhaustive study would require much more space than is available here, and the reader is referred to Friend ${ }^{14}$ and Moschytz ${ }^{15}$ for two important circuits. However a further two types of circuit will be mentioned in the context of this paper. Within the reported work neither of these could be regarded as a panacea; it is believed however that they are of importance. Another concept which will not be discussed here is the "leapfrog" structure ${ }^{16}$ as it would seem to require a large number of amplifiers and not offer any advantage over the P.I.C. technique, for lowpass circuits.

\subsection{Two Capacitor One Amplifier Zero Forming Circuits}

A number of circuits are available, capable of forming the general lowpass stage with a transmission zero

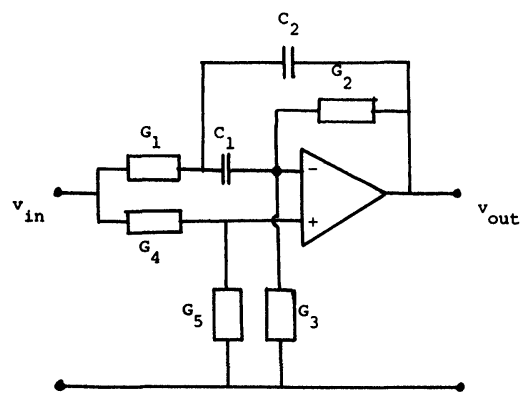

FIGURE 19 Two capacitor one amplifier circuit with transmission zeros.

${ }^{\dagger}$ However some filters require unequal source and load. In this case, the relative matching still needs to be maintained. and one such circuit is shown in Figure 19. ${ }^{17}$ The transmission function for this circuit is

$T(s)=\frac{G_{4}}{G_{4}+G_{5}}\left[\frac{s^{2}+s\left[\left(G_{2}+G_{3}\right)\left(\frac{1}{C_{2}}+\frac{1}{C_{1}}\right)-\frac{G_{5}}{G_{4}} \frac{G_{1}}{C_{2}}\right]+\frac{G_{1}\left(G_{2}+G_{3}\right)}{C_{1} C_{2}}}{s^{2}+s G_{2}\left(\frac{1}{C_{2}}+\frac{1}{C_{1}}\right)+\frac{G_{1} G_{2}}{C_{1} C_{2}}}\right.$

for which

$$
\begin{aligned}
\omega_{r} & =\sqrt{\frac{G_{1} G_{2}}{C_{1} C_{2}}} \\
Q & =\sqrt{\frac{G_{1}}{G_{2}}} \frac{1}{\sqrt{\frac{C_{1}}{C_{2}}},+\sqrt{\frac{C_{2}}{C_{1}}}}
\end{aligned}
$$

and if

$$
\left(G_{2}+G_{3}\right)\left(\frac{1}{C_{2}}+\frac{1}{C_{1}}\right)=\frac{G_{5} G_{1}}{G_{4} C_{2}}
$$

then a perfect zero exists at

$$
\omega_{\infty}=\sqrt{\frac{G_{1}\left(G_{2}+G_{3}\right)}{C_{1} C_{2}}}
$$

As far as is known no circuit of this type allows for non-iterative tuning, nor have they been reported as being used successfully in reasonably demanding situations. However as in the reference quoted they may prove of use in low $Q$ circuits, when cascaded with sections of a different type.

\subsection{Active R Circuits}

Over recent years development of a number of so called active $R$ circuits have been reported, ${ }^{18}$ a typical circuit of which is shown in Figure 20. The

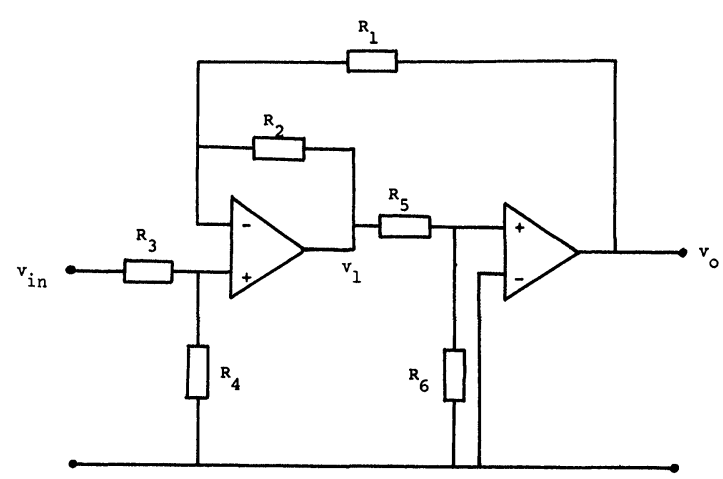

FIGURE 20 Active $\mathrm{R}$ circuit. 
basis of these designs is to use an operational amplifier well above its cut-off frequency.

As if

$$
A=\frac{A_{0}}{1+s / s_{0}}
$$

then for

$$
s \gg s_{0} A \sim \frac{A_{0} s_{0}}{s}
$$

and if $A_{0} s_{0}$ is stabilised, then the amplifier behaves as a near ideal integrator and be used in a manner analogous to the state variable circuit. For the circuit in Figure 20 the transfer function is

$$
\frac{v_{0}}{v_{\text {in }}}=\frac{G B^{2} \frac{R_{4}}{R_{3}+R_{4}} \frac{R_{6}}{R_{5}+R_{6}}}{s^{2}+G B \frac{R_{1}}{R_{1}+R_{2}} s+G B^{2} \frac{R_{2}}{R_{1}+R_{2}} \frac{R_{6}}{R_{5}+R_{6}}}
$$

and

$$
\frac{v_{1}}{v_{\text {in }}}=\frac{G B . s . \frac{R_{4}}{R_{3}+R_{4}}}{s^{2}+\frac{G B R_{1}}{R_{1}+R_{2}} s+G B^{2} \frac{R_{2}}{R_{1}+R_{2}} \frac{R_{6}}{R_{5}+R_{6}}}
$$

there

$$
G B=A_{0} s_{0}
$$

thus

$$
\begin{gathered}
\omega_{r}=G B \sqrt{\frac{R_{2}}{R_{1}+R_{2}} \frac{R_{6}}{R_{5}+R_{6}}} \\
Q=\sqrt{\left(1+\frac{R_{2}}{R_{1}}\right) \frac{R_{2}}{R_{1}} \frac{R_{6}}{R_{5}+R_{6}}}
\end{gathered}
$$

Now for commonly used amplifiers $G B \sim 2 \pi 10^{6}$ and typical parameters for a lowpass filter are $\omega_{r} \sim 2 \pi 10^{3}$ and $Q \sim 5$.

$$
\therefore \frac{R_{6}}{R_{5}+R_{6}} \sim 10^{-6}
$$

and

$$
\frac{R_{2}}{R_{1}} \sim 5.10^{3}
$$

These resistor ratios are inconveniently large and for this reason active $R$ filters have been proposed for use at frequencies close on the gain bandwidth product.
Also at the frequency suggested above the approximation that the amplifier is a good integrator is not necessarily valid, and the lowpass circuit will not behave exactly as predicted when the fact that the integrator pole is not precisely at $s=0+j 0$ is considered. However it could well be that in the not too distant future integrated amplifiers with the open loop pole at a lower frequency, and hence a lower gain bandwidth product, could become available, thus making this technique viable at lower frequencies. It would be necessary for the gain bandwidth product to be stable to a high degree.

\section{AMPLIFIER LIMITATIONS}

The main objective of this note is to discuss the problems of assembling active filter circuits using hybrid technology; however for the sake of completeness some mention of the limitations imposed by the active components will be made and some comparisons with the effects of passive component variations presented.

When circuits are to be constructed using hybrid technology it is often considered valid to assume that the resistors will drift together and will have similar TCR's. This is usually a reasonable assumption provided all resistors have been subjected to the same processes. However often resistors are made from different materials (i.e. different sheet resistivities) and occasionally a resistor ratio is set using just one resistor (e.g. when setting the amplifier gain in the Sallen and Key equal capacitor circuit) and under these circumstances the assumption that component values track needs re-examining.

Moschytz ${ }^{21}$ has used the assumption that component values track and has demonstrated that the most significant variable is the amplifier open loop gain and has derived a figure of merit, gain sensitivity product (GS), where

$$
G S=K . S_{K}^{Q}
$$

Using this criterion the Sallen and Key, and Lim circuits have $G S \sim Q$ and for the Rauch circuit $G S \sim Q^{2}$. This analysis also assumes that the open loop gain is purely real, whereas for commonly used amplifiers at frequencies of general interest, there is a significant phase shift.

To demonstrate the effect of a "real" amplifier four filter circuits have been compared under various assumptions. These are detailed in Table III together with a reference to the graph which shows the transfer function. 
82

P. L. MORAN
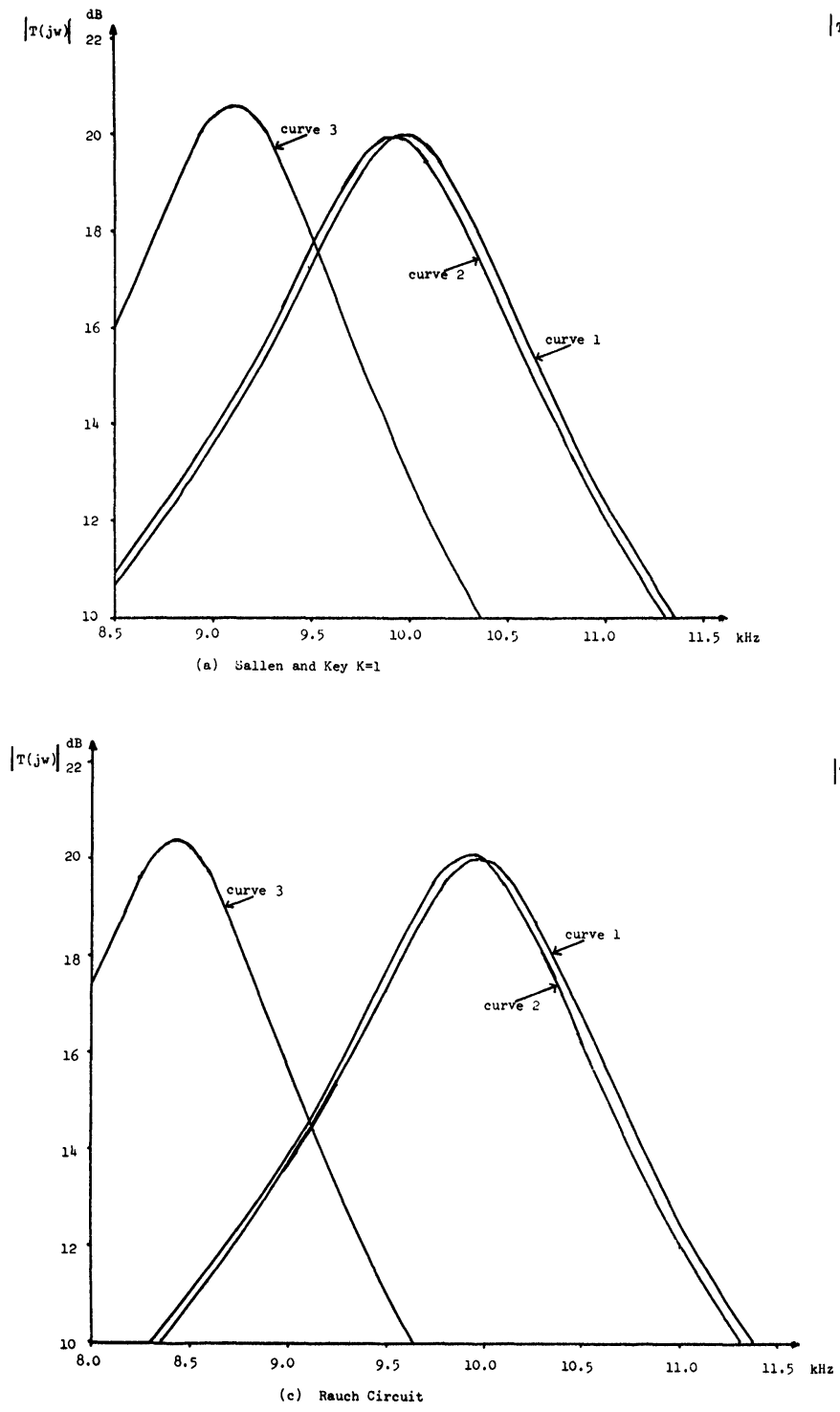
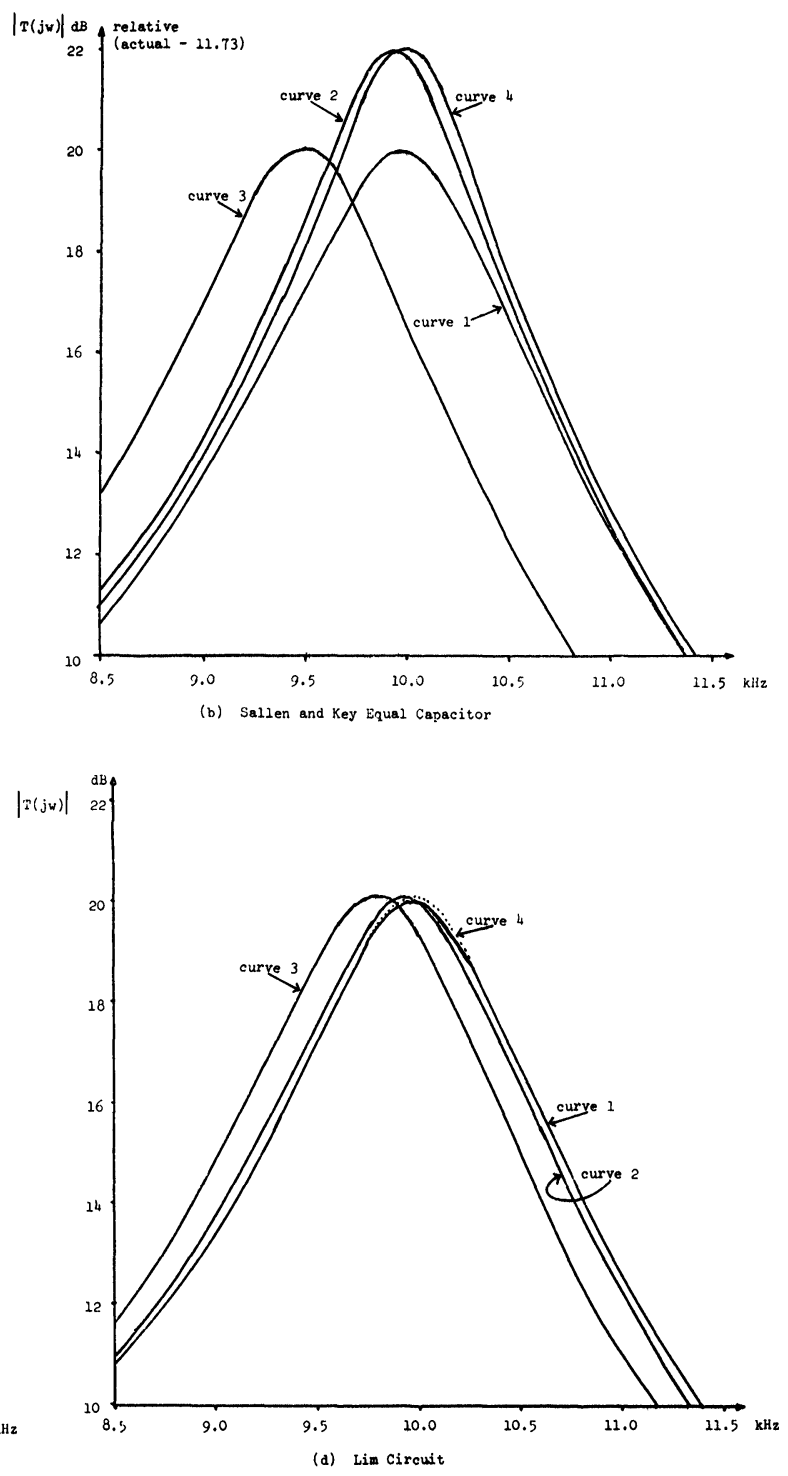

FIGURE 21 Comparison of various active filter circuits with respect to component variations and amplifier performance.

Each circuit has been designed for a $Q$ of 10 and $f_{0}$ of $10 \mathrm{kHz}$. The equal capacitor circuits all use $1 \mathrm{nF}$ capacitors and in the others, the resistors are in the region of $10 \mathrm{k} \Omega$. It can be seen that the Rauch circuit has good tolerance to passive component variation but is highly sensitive to the amplifier characteristics. The Galen and Key circuits are rather better than the Rauch circuit in this respect and the equal capacitor circuit is rather less sensitive than the unity gain circuit, but it can also be seen that the equal capacitor circuit is comparatively very sensitive to passive component changes. The $\mathrm{Lim}$ circuit has the best performance with respect to amplifier limitations and also is fairly insensitive to passive component variations.

The positive impedance converter circuit is invariably used in a simulated LC ladder and the structure of this type of filter is itself of very low sensitivity. The effective value of the transformed element will of course be subject to variations in the passive components. By proper design and trimming however it is possible to overcome the deleterious 
TABLE III

Comparison of four filter circuits.

\begin{tabular}{|c|c|c|c|c|}
\hline & $\begin{array}{l}\text { Sallen and Key } \\
K=1\end{array}$ & $\begin{array}{l}\text { Sallen and Key } \\
\text { equal } C\end{array}$ & Rauch & Lim \\
\hline Ideal components & $\begin{array}{l}\text { Fig. } 21 \mathrm{a} \\
\text { curve } 1\end{array}$ & $\begin{array}{l}\text { Fig. } 21 \mathrm{~b} \\
\text { curve } 1\end{array}$ & $\begin{array}{l}\text { Fig. } 21 \mathrm{c} \\
\text { curve } 1\end{array}$ & $\begin{array}{l}\text { Fig. } 21 \mathrm{~d} \\
\text { curve } 1\end{array}$ \\
\hline $\begin{array}{l}1 \% \text { variation in a } \\
\text { passive component. } \\
\text { Ideal amplifiers. }\end{array}$ & $\begin{array}{l}\text { Fig. 21a } \\
\text { curve } 2\end{array}$ & $\begin{array}{l}\text { Fig. } 21 b \\
\text { curve } 2\end{array}$ & $\begin{array}{l}\text { Fig. } 21 \mathrm{c} \\
\text { curve } 2\end{array}$ & $\begin{array}{l}\text { Fig. } 21 d \\
\text { curve } 2\end{array}$ \\
\hline $\begin{array}{l}\text { Ideal passive components } \\
\text { Amplifier gain }=10^{5} \\
\text { Amplifier bandwidth } 10 \mathrm{~Hz}\end{array}$ & $\begin{array}{l}\text { Fig. } 21 \mathrm{a} \\
\text { curve } 3\end{array}$ & $\begin{array}{l}\text { Fig. } 21 \mathrm{~b} \\
\text { curve } 3\end{array}$ & $\begin{array}{l}\text { Fig. } 21 \mathrm{c} \\
\text { curve } 3\end{array}$ & $\begin{array}{l}\text { Fig. } 21 \mathrm{~d} \\
\text { curve } 3\end{array}$ \\
\hline $\begin{array}{l}\text { Ideal amplifier } \\
1 \% \text { change in a gain } \\
\text { determining resistor. }\end{array}$ & & $\begin{array}{l}\text { Fig. } 21 \mathrm{~b} \\
\text { curve } 4 \\
1 \% \text { change in } K\end{array}$ & & $\begin{array}{l}\text { Fig. } 21 \mathrm{~d} \\
\text { curve } 4 \\
1 \% \text { change } \\
\text { in } R 5\end{array}$ \\
\hline
\end{tabular}

TABLE IV

Advantages and disadvantages of various filter types.

\begin{tabular}{|c|c|c|}
\hline & Advantages & Disadvantages \\
\hline $\begin{array}{l}\text { Sallen and Key } \\
K=1\end{array}$ & low sensitivity & $\begin{array}{l}\text { wide capacitor spread } \\
\text { no zeros } \\
\text { not trimmable }\end{array}$ \\
\hline $\begin{array}{l}\text { Sallen and Key } \\
\text { Equal capacitors }\end{array}$ & $\begin{array}{l}\text { equal capacitors } \\
\text { trimmable }\end{array}$ & $\begin{array}{l}\text { poor sensitivity } \\
\text { no zeros }\end{array}$ \\
\hline Rauch & $\begin{array}{l}\text { low sensitivity to } \\
\text { passive components }\end{array}$ & $\begin{array}{l}\text { wide capacitor spread } \\
\text { not trimmable } \\
\text { no zeros }\end{array}$ \\
\hline State variable & $\begin{array}{l}\text { equal capacitors } \\
\text { trimmable } \\
\text { zeros }\end{array}$ & $\begin{array}{l}\text { large number of } \\
\text { amplifiers }\end{array}$ \\
\hline Lim & $\begin{array}{l}\text { equal or near equal } \\
\text { capacitors } \\
\text { trimmable } \\
\text { zeros } \\
\text { low sensitivity }\end{array}$ & $\begin{array}{l}\text { large number of } \\
\text { capacitors } \\
\text { trim requires } \\
\text { sub-network formation }\end{array}$ \\
\hline P.I.C. & $\begin{array}{l}\text { low sensitivity } \\
\text { equal capacitors } \\
\text { trimmable when zeros } \\
\text { are included } \\
\text { few active trims }\end{array}$ & $\begin{array}{l}\text { large number of } \\
\text { amplifiers }\end{array}$ \\
\hline
\end{tabular}


effects of the amplifier and to arrive at components with a very high $Q$ factor at the frequency of interest. Moreover provided the amplifier gain-bandwidth products track as a function of time, temperature etc. the circuit will retain its high $Q$ factor. Obviously good tracking is best achieved by using dual amplifier chips. An excellent discussion of the P.I.C. is given by Saraga, Haigh and Barker. ${ }^{19}$

\section{SUMMARY AND CONCLUSION}

To attempt to compare the various active filter circuits without having an application in mind is impossible. Similarly the environment in which the filter will be used is equally important. The marginal advantage is saving one capacitor when the circuit is hermetically packaged is questionable, particularly if it results in a more sensitive circuit. For comparison purposes therefore it will be assumed that the packaging is such that the complexity of the circuit is important and also that specification of the filter is sufficiently demanding that functional adjustment is necessary (i.e. adjustment by measuring the capacitor values and calculating the resistor values is not adequate). Such a filter could be the specification demanding an eight pole $0.1 \mathrm{~dB}$ Tchebychev or five pole four zero elliptic (if the out of band attenuation does not have to tend rapidly to infinity then both these functions can be considered as having similar cut off characteristics).

The filters will be compared both by a "costing" that assigns relative values to capacitors, amplifiers and functional trims and by a table that outlines the advantages and disadvantages of each. These comparisons are given in Tables IV and V. The approximate relative costings need treating with some caution since they are liable to change quite dramatically, and since assembly costs will vary with the manufacturing technique used.

The amplifier "costs" are for an unencapsulated amplifier and the capacitor costs for $10 \%$ tolerance high stability "chips". It is assumed that the marginal costs of extra resistors and passive trimming is negligible. Four "costing" functions have been assumed based on Table VI.

TABLE VI

Costing functions.

\begin{tabular}{llll}
\hline & Amplifier & Capacitor & Active Trim \\
\hline$C_{1}$ & 1 & 1 & 1 \\
$C_{2}$ & $1 \frac{1}{2}$ & 1 & 1 \\
$C_{3}$ & 1 & 1 & $\frac{1}{2}$ \\
$C_{4}$ & $1 \frac{1}{2}$ & 1 & $\frac{1}{2}$ \\
\hline
\end{tabular}

Only those filter types capable of functional trimming have been "costed".

From these tables it can be seen that elliptic filter sections provide a more cost effective solution if this type of filter function can be used and of these, the P.I.C. technique is probably the most useful. It is worth bearing in mind that the equipment necessary to trim the P.I.C. is more complex than for the other

TABLE V

Component count and "costings" for various filter functions.

$T=8$ pole $T$ chebychev

$E=5$ pole 4 zero elliptic

\begin{tabular}{llllllllll}
\hline & Function & $\begin{array}{l}\text { no. of } \\
\text { capacitors }\end{array}$ & $\begin{array}{l}\text { no. of } \\
\text { amplifiers }\end{array}$ & $\begin{array}{l}\text { no. of } \\
\text { active trims }\end{array}$ & d.c. gain & $C_{1}$ & $\mathrm{C}_{2}$ & $C_{3}$ & $C_{4}$ \\
\hline $\begin{array}{l}\text { Sallen and Key } \\
\text { equal capacitors }\end{array}$ & $T$ & 8 & 4 & 8 & fixed & 20 & 22 & 16 & 18 \\
State variable & $E$ & 5 & 7 & 5 & variable & 17 & 21 & 15 & 18 \\
State variable & $T$ & 8 & 12 & 8 & variable & 28 & 34 & 24 & 30 \\
Lim & $E$ & 9 & 3 & 9 & variable & 21 & 23 & 17 & 18 \\
Lim & $T$ & 12 & 4 & 12 & fixed & 28 & 30 & 22 & 24 \\
P.I.C. & $E$ & 6 & 6 & 2 & variable & 14 & 17 & 13 & 16 \\
\hline
\end{tabular}




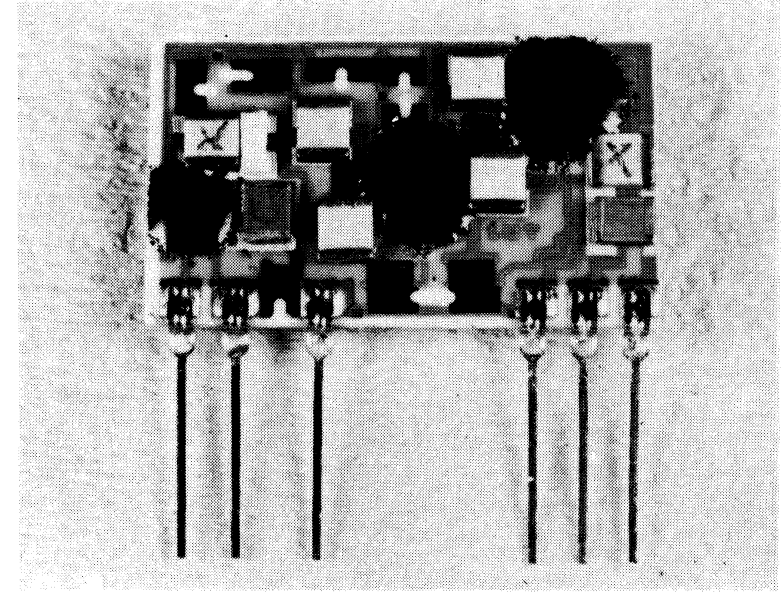

FIGURE 22 Thick film implementation of the five pole four zero elliptic filter using the P.I.C. circuit of Figure 19 (note this includes power supply decoupling capacitors).

types since the trim is carried out by placing a voltage transfer minimum at the correct frequency rather than setting a specific voltage gain or phase, although it may be possible in some applications to say that when the gain is below some fixed figure the minimum is correctly placed. If the application necessitates the use of a Tchebychev or Butterworth type of function then, provided the relatively high sensitivity can be tolerated the Sallen and Key equal capacitor circuit appears cost effective.

Figure 22 shows a thick film implementation of the five pole four zero elliptic filter using the positive impedance converter techniques.

\section{REFERENCES}

1. R. Sallen and E. Kay "A practical method of designing RC active filters" Trans. IRE Circuit Theory. CT2 (March 1955) pp. 74-85.

2. A. B. Williams Active Filter Design (Artech House Inc. Massachusetts 1975).

3. H. Orchard "Inductorless filters" Electron. Lett. 2, 6 (June 1966) pp. 224-5.

4. F. F. Kuo Network Analysis and Synthesis section 14.6 (Wiley)
5. A. Antoniou "Realisation of gyrators using operational amplifiers and their use in RC active network synthesis" Proc IEEE. 116, 11 (Nov 1969) pp. 1838-1850.

6. G. C. Temes and S. K. Mitra Modern Filter Theory and Design (Wiley 1973).

7. J. T. Lim "Improvements in or relating to active filter networks" British Patent No. 1383204 (filed 16.4.71).

8. A. Sedra "A class of stable active filters using unity gain voltage followers" IEEE J. Solid-State Circuits. SC7, 1, (1972) pp. 311-315.

9. G. S. Moschytz "A general approach to Twin T design and its application to hybrid integrated linear active networks" Bell System Technical Journal. (July-August 1970) pp. 1105-1149.

10. A. Hastings "Analy sis of a resistance-capacitance parallel-T network and its applications" Proc IRE. 3, (March 1946) pp. 126-129.

11. G. S. Moschytz "Two step precision tuning of Twin T notch filter" Proc IEEE. 54, 5 (May 1966) pp. 811-812.

12. R. Jeffers and D. Haigh "Active RC lowpass filters for fdm and pcm systems" Proc IEEE. 120, 9 (1973) pp. 945-953.

13. L. T. Bruton "Network transfer functions using the concept of frequency dependent negative resistance" IEEE Trans. Circuit Theory. CT16 (August 1969) pp. 406-8.

14. J. J. Friend et al "Star - an active biquadratic filter section" IEEE Trans. Circuits and Syst. CAS22 (1975) pp. $115-120$.

15. G. S. Moschytz "Fen filter design using tantalum and silicon integrated circuits" Proc IEEE. 58 (1970) pp. 550-565.

16. F. E. J. Girling and E. F. Good "The leapfrog or active ladder synthesis" Wireless World. 76 (July 1970) pp. $341-5$.

17. Y. Katsu ta and K. Suzuki "Thick film RC active filters for PCM applications" Proc ISHM Conf. 1978 Minneapolis pp. 344-348 and Proc IEEE. 67, 1 (Jan 1979) pp. 34-37.

18. J. R. Brand and R. Schaumann "Active R filters: a review of theory and practice" IEEE J. Electronic Circuits and Syst. 2, 4 (July 1978).

19. W. Saraga, D. Haigh and R. Barker "A design philosophy for microelectronic active RC filters" Proc IEEE. 67, 1 (Jan 1979) pp. 24-33.

20. W. Saraga, D. Haigh and R. Barker "Microelectronic active $\mathrm{RC}$ channel bandpass filters in the frequency range $60-108 \mathrm{kHz}$ for FDM SSB telephone systems" IEEE Trans. Circuits and Syst. CAS25, 12 (Dec 1978) pp. 1022-1031.

21. G. S. Moschytz "Gain-Sensitivity Product - A figure of merit for Hybrid Integrated Filters using single operational amplifiers" IEEE J. Solid State Circuits. SC6, 3 (June 1971) pp. 103-110. 

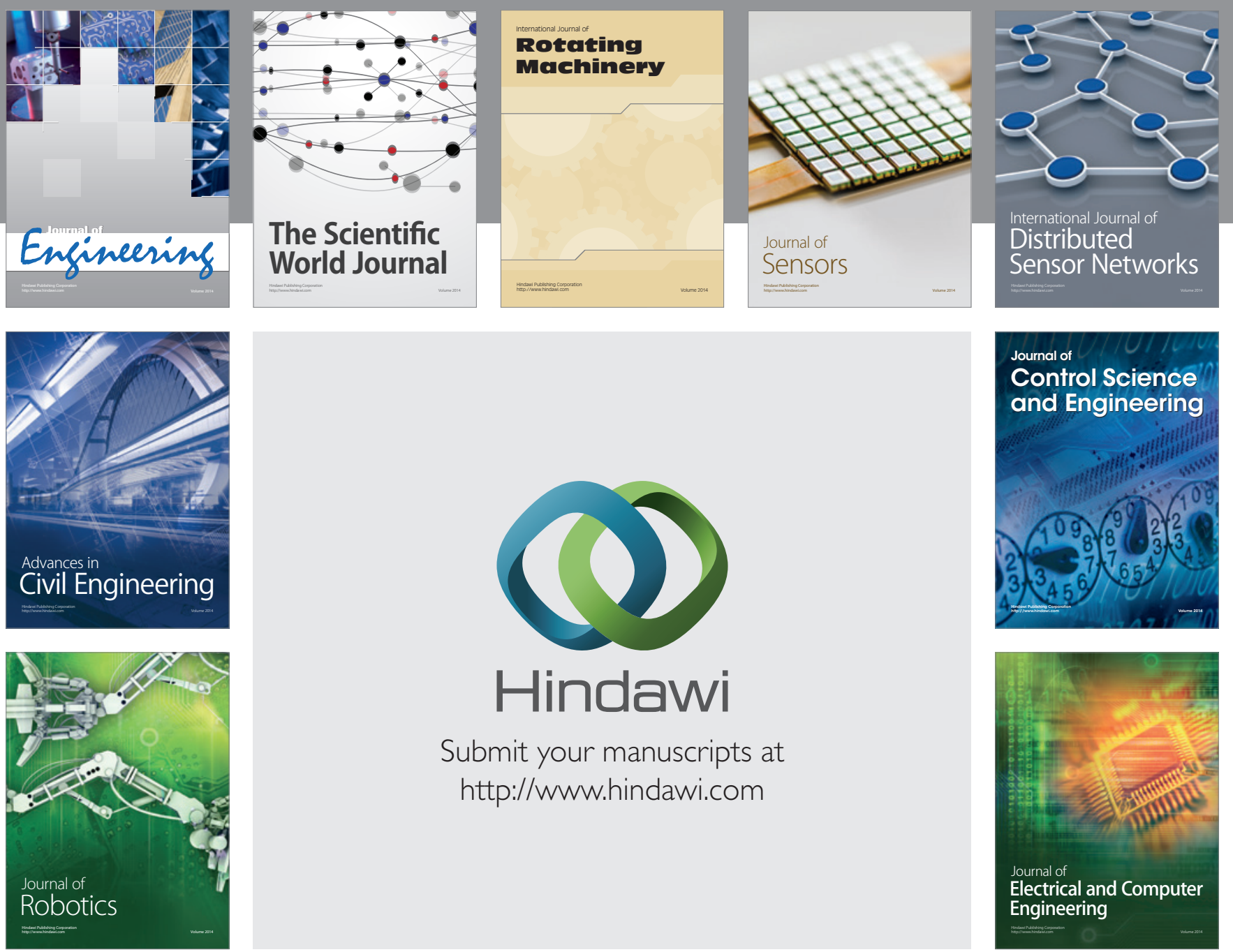

Submit your manuscripts at

http://www.hindawi.com
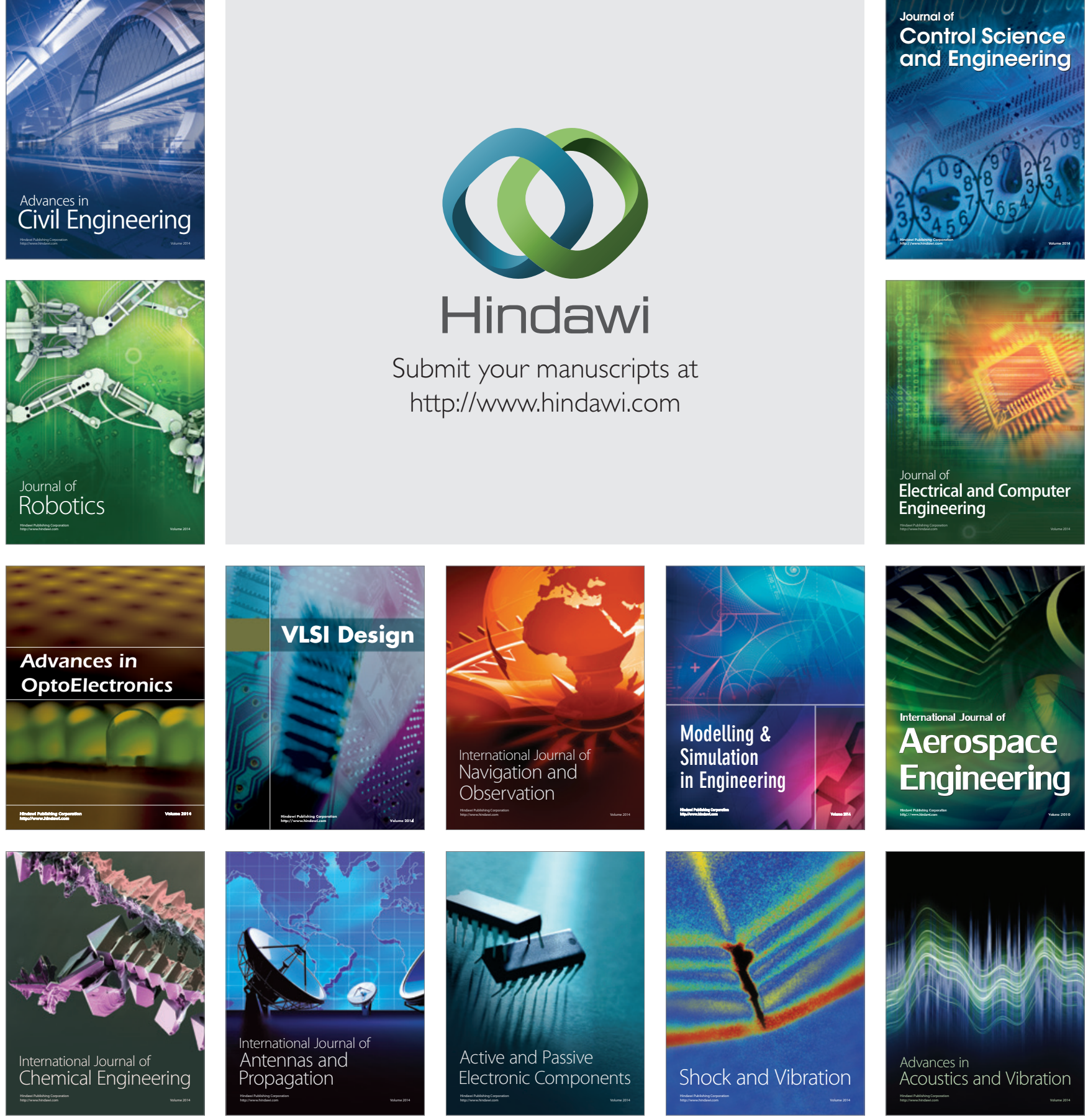